\title{
Extracting Information from Adaptive Control Experiments
}

\author{
James L. White, ${ }^{[a]}$ Elizabeth C. Carroll ${ }^{[\mathrm{b}]}$ Kenneth G. Spears, ${ }^{[\mathrm{c}]}$ and Roseanne J. Sension $*[\mathrm{c}]$
}

Abstract: Optical control of chemical reactivity is achieved through the use of photonic reagents, that is, "shaped" ultrafast optical pulses created using a pulse shaper. It has been demonstrated in a number of molecular systems that these pulses can effectively guide the system into a desired final state. Effective pulses are often found through an experimental search involving thousands of individual measurements. An examination of the pulses tested in these ex- periments can reveal the pulse features responsible for control and also the underlying molecular dynamics. In this article we review attempts to extract information from optical control experiments using adaptive learning algorithms to search the available parameter space, and we discuss how these kinds of experiments can be used to achieve and understand multiphoton optical control.

Keywords: laser chemistry · learning algorithms · optical control · photophysics · ultrafast chemistry

\section{Introduction}

In the study of photochemical reactions laser pulses serve as both reagent and measuring device. The role of measuring device has been exemplified by ultrafast spectroscopies that exploit broadband optical pulses to probe molecular dynamics with femtosecond time resolution. More sophisticated multi-dimensional spectroscopies are using optical pulse-shaping to take measurement of molecular level quantum coherence to a new level of sensitivity and selectivity. ${ }^{[1-4]}$ The role for laser pulses as reagents is less explored, but has the potential to address one of the fundamental goals of chemistry: control of chemical reactivity. While in most spectroscopic studies the role of the incident "pump" pulse is limited to that of a tunable energy source, the field of optical coherent control focuses on designing optical fields that will drive the light-matter interaction toward a particular chemical outcome. Owing to over a decade of technical advances in the development of broadband lasers and optical manipulation, it is now possible to create complex pulses in the laboratory with commercial pulse shapers. A typical scheme using a spatial light modulator or acousto-optic modulator to manipulate broadband optical pulses is illustrated in Figure 1. These shaped pulses may be used as smart reagents, ${ }^{[5-8]}$ or smart catalysts, ${ }^{[9]}$ to control molecular dynamics and chemical reactivity by producing specific quantum states or by steering a molecule through a series of light-matter interactions.

The majority of optical control experiments have used shaped visible or near-IR pulses. The capability for optical pulse shaping has recently been extended to the ultraviolet region of the spectrum using an acousto-optic mod- ulator in a 4-f arrangement (Figure 1) ${ }^{[10-12]}$ an acoustooptic programmable dispersive filter (AOPDF, Dazzler, Fastlite) ${ }^{[13,14]}$ or a microelectromechanical system (MEMS) array. ${ }^{[15,16]}$ The ability to manipulate and characterize ultraviolet pulses greatly extends the range of chemical systems available for spectroscopic investigation and control using shaped pulses. Many important small molecule systems absorb between 200 and $400 \mathrm{~nm}$, and may now be studied, including both cyclohexadiene ringopening reactions and cis-stilbene isomerization reactions. $^{[11,14,17]}$

Identifying shaped optical pulses that will act as chemical reagents is a challenge. The space of potential pulse shapes - potential reagents - is enormous, and the identification of optimally effective reagents or catalysts

[a] J. L. White

Department of Applied Physics, Stanford University 348 Via Pueblo Mall

Stanford, CA 94305-4090, USA

[b] E. C. Carroll

Department of Molecular and Cell Biology

University of California, Berkeley

142 Life Sciences Addition \# 3200

Berkeley, CA 94720-3200, USA

[c] K. G. Spears, R. J. Sension

Department of Chemistry, University of Michigan

930 N. University Ave.

Ann Arbor, MI 48109-1055 USA

phone: $+1734763-6074$

fax: + 1734 647-4865

e-mail: rsension@umich.edu 
poses a significant problem. Computational methods are at present insufficient to identify appropriate pulse shapes for all but the simplest of physical systems. If physical intuition can be used to sufficiently limit the search space, a systematic search can be used to identify

James L. White received his bachelor's degree in Physics and Mathematics from the University of Michigan and is currently a graduate student in Applied Physics at Stanford University. His research focuses on the use of statistical methods to analyze data sets obtained in optical control experiments and to use this analysis to extract mechanistic insight.

Elizabeth C. Carroll received her B.S. in Physics from Gonzaga University in Spokane, Washington, and her Ph.D. in Applied Physics from the University of Michigan in 2006 for work on coherent control in ultrafast solution phase chemistry. She performed postdoctoral research at the University of California, Davis and is now a postdoctoral fellow at the University of California, Berkeley.

Kenneth G. Spears is an Emeritus Professor of Chemistry from Northwestern University now working with Professor Sension at the University of Michigan. He received his Ph.D. from The University of Chicago with Stuart Rice in 1970 and retired from Northwestern in August, 2009. Professor Rice has been a great inspiration in his work, and he congratulates him on his award of the Wolf Prize for Chemistry

Roseanne J. Sension is Professor of Chemistry and Physics at the University of Michigan. She received her bachelor's degree in Chemistry and Mathematics from Bethel University in St. Paul in 1981 and her Ph.D. in Chemistry from the University of California, Berkeley, in 1986. She has been on the faculty at Michigan since 1992. Her research interests include the use of ultrafast lasers and spectroscopic techniques to observe and control photoinitiated reactions.
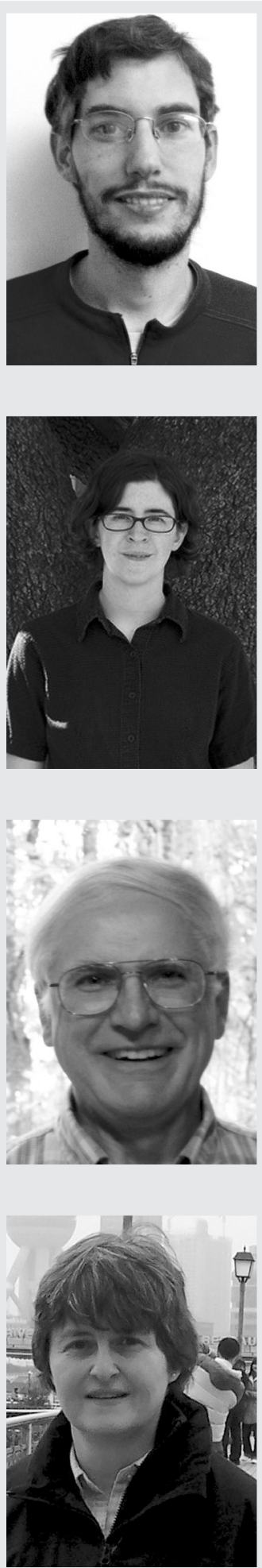

\section{Adaptive Control Concepts}

\subsection{Learning Algorithms and the Search for Optimal Pulses}

Optical control of complex reactive systems begins with the identification of fields capable of achieving the desired target state. Quantum optimal control theory ${ }^{[23]}$ allows the calculation of optimal pulses by propagation of wave packets under the Hamiltonian of a test field. However, even a molecule as small as cyclohexadiene, with 36 vibrational degrees of freedom, is large enough to prohibit sufficiently accurate electronic structure calculations. When the influence of a solvent bath and decoherence are included, the problem is computationally intractable by current methods. Instead, it is possible to approach the problem empirically with an experimental search for effective pulse shapes. Since the size of the optical parameter space typically precludes an exhaustive experimental search, an iterative search algorithm incorporating experimental feedback is used instead. ${ }^{[18,21,24]}$ The effect of a given field on the system of interest is evaluated experi- 
mentally, and then the algorithm uses this information to identify further candidate fields for evaluation.

Following a suggestion by Judson and Rabitz, ${ }^{[18]}$ genetic algorithms incorporating experimental feedback have become widely used to search for control fields. The genetic algorithm (GA) is a search and optimization strategy inspired by biological evolution. Although there is no guarantee that a GA will identify a globally optimal pulse for control, GAs have proven remarkably able to identify nontrivial control fields in a wide variety of physical systems. ${ }^{[10,20,21,25]}$ Inside the algorithm, each optical pulse is represented as a string of numbers (a genome). In our experiments, pulse genomes typically consist of spectral phase and/or amplitude as a function of wavelength. ${ }^{[22]}$ The algorithm begins with a random initial collection of pulses (a population). Each pulse in the population is tested against the control objective and assigned a merit score, or fitness. In a typical experiment, this evaluation step involves using a computer-controlled pulse shaper to physically create the pulse encoded by the genome, having the pulse interact with the system under investigation, and then measuring some effect such as a change in absorption. After the entire population of pulses has been evaluated, mating and mutation operators are applied to the genomes of the higher-merit pulses to produce a new generation of pulses. Genomes with high fitness tend to encode desirable pulse traits, and these traits eventually dominate the population. A refinement of the GA involves the inclusion of an adaptive learning step that evaluates the relative success of the various mating and mutation operators at improving the fitness of the each generation and weights the use of each operator according to its past accomplishment. ${ }^{[22]}$

The choice of basis for performing the search (the representation used for genomes) and the choice of basis for performing any analysis are important. ${ }^{[26]}$ The choice of search space parameterization affects the ease with which solutions are found and even which solutions are found..$^{[27,28]}$ Theory, simulation, and experiment have all shown that in general there exist distinct solutions that perform equally well. ${ }^{[28-30]}$ The influence of basis set and parameterization on experimental outcomes and the presence of multiple solutions complicate efforts to extract physical insight into control mechanisms from adaptive control experiments.

\subsection{Coherent Control Mechanisms}

Optical control over molecular dynamics using learning algorithms and shaped ultrafast laser pulses has been realized experimentally in many forms, ${ }^{[5-8]}$ including strongfield multiphoton control of molecular rearrangements in the gas phase, ${ }^{[20,21,26,31-40]}$ control of excited-state populations in resonant transitions in liquids, ${ }^{[25,41-51]}$ and limited control of condensed phase isomerization reactions. ${ }^{[52-55]}$ Literature discussions of coherent control mecha- nisms ${ }^{[56-60]}$ typically invoke one or more of the three processes illustrated schematically in Figure 2: (a) interfering multiphoton pathways, (b) "pump-dump" sequences, and (c) wavelength tuning.

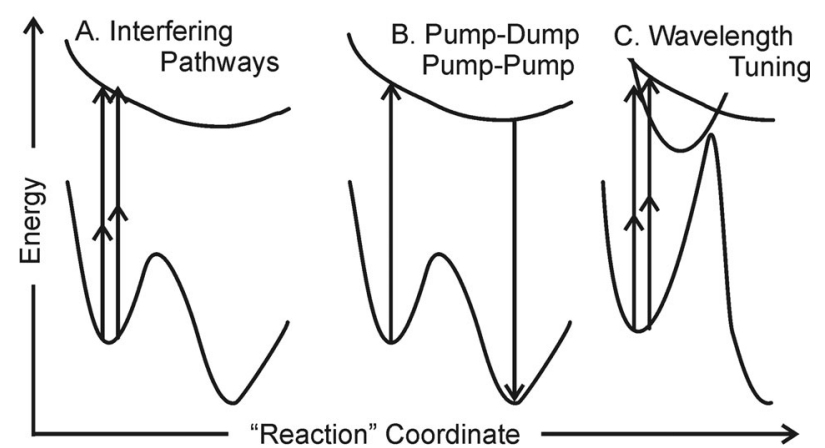

Figure 2. Cartoon illustrating three mechanisms for multiphoton control of chemical dynamics.

The theory for control mechanisms involving multiple pathway interference (Figure $2 \mathrm{a}$ ) has been developed by Brumer and Shapiro. ${ }^{[58,59,61]}$ The high density of states present in molecules and the ability of the optical field to excite various transitions lead to a large number of possible paths between the initial and final states. The driving laser field is coherent and so excites a coherent set of quantum pathways. These pathways then interfere, resulting in a molecular version of Young's double-slit experiment. Manipulating the driving field changes the interferences and thus the outcome. ${ }^{[62]}$

Figure 2 b illustrates the "pump-dump" mechanism described extensively by Tannor and Rice. ${ }^{[60,63]}$ Conceptually, pump-dump uses the timing between sequential interactions to direct the temporal evolution of a wave packet. In the simplest form a sequence of precisely timed interactions is used to manipulate the electronic state population and wave packet dynamics. Although this mechanism is often called a pump-dump mechanism, as the most common interactions involve cycling between the ground and excited electronic state, the mechanism can also involve excitations to higher excited electronic states. In one particularly interesting recent example, multiphoton pump-repump schemes were exploited to distinguish nearly identical molecules. ${ }^{[10,64,65]}$ Small variations in the dynamics allow optimal dynamic discrimination (ODD) of molecules with nearly indistinguishable optical properties.

Multiphoton interference within the optical field may enhance or suppress the multiphoton transition probability for a given transition via shaping the nonlinear power spectrum of the pulse (discussed in more detail below). ${ }^{[66-69]}$ This mechanism is illustrated in Figure $2 \mathrm{c}$. Although this third mechanism is not a molecular quantum control mechanism, since the "control" is entirely 
confined to the classical radiation field, it must be taken into account any time the control process involves multiphoton excitation. Excitation control achieved through tuning multiphoton transition probabilities has been exploited to selectively excite spectrally overlapping dye molecules in solution, ${ }^{[43]}$ excite fluorescent probes in living systems while suppressing autofluorescence, ${ }^{[68]}$ and to optimize different orders of multiphoton absorption. ${ }^{[67,69]}$ Under the formalism of Brixner et al. ${ }^{[70]}$ spectral control via the two-photon absorption (TPA) spectrum of the target state was recently identified by Ogilvie et al. ${ }^{[71]}$ and by Montgomery and Damrauer. ${ }^{[49]}$

\section{Identifying Control Parameters in Multidimensional Search Sets}

During each GA search, hundreds or thousands of different pulses shapes are evaluated. Systematic analysis of correlations between pulse characteristics and pulse performance for all of the pulses in the search set ${ }^{[72,73]}$ has the potential to reduce the multidimensional genome space to a minimal set of control parameters. ${ }^{[38,52,73-76]}$ Intuitive parameter reduction is guided by a phenomenological hypothesis: if a parameter $\mathrm{X}$ is highly correlated with fitness, then parameter $\mathrm{X}$ is a control parameter. This statement holds true in the simple case of optimizing second harmonic generation: $\mathrm{SHG}$ is known to depend on $\mathrm{I}^{2}$, where $\mathrm{I}(\mathrm{t})$ is the intensity of the optical pulse, thus $\mathrm{I}^{2}$ is correlated with fitness. SHG depends on the magnitude of linear chirp, but not the sign. Fitness is observed to be highly correlated with $\left|\phi^{\prime \prime}\left(\omega_{0}\right)\right|$, but is uncorrelated with the sign of chirp.

Even processes as simple as two-photon absorption or SHG, however, introduce complications into the direct correlation of a specific parameter with fitness. When the pulse is sufficiently intense the transform-limited pulses with the maximum peak intensity may not provide a unique or optimal solution to a search for maximum SHG. ${ }^{[2]}$ Recent work has concentrated on elucidation of the nature of fitness landscapes using two photon absorption and second harmonic generation as test cases. ${ }^{[27,29,77-79]}$

More formally, several strategies based on covariance analysis have been demonstrated recently to determine minimal sets of control parameters in complex reactive systems, including applications of principle component analysis (PCA) (dubbed Principle Control Analysis by White et al.), ${ }^{[73]}$ and partial least squares regression (PLS) analysis by Montgomery et al. ${ }^{[72,75]}$ Before discussing these multivariate techniques, we review single-parameter controls.

\subsection{Intuitive Parameter Reduction}

\subsubsection{Pulse Intensity and Nonlinear Power Spectrum}

Nonlinear power spectra provide an intuitive way to consider the effect of shaped pulses on multiphoton transitions. The probability of $n$-photon absorption at $\omega$ is proportional to the nonlinear power spectrum $\mathrm{S}_{n}(\omega)$ that results from summing the contributions of all photon $n$ tuples in the pulse bandwidth whose total energy sums to $\omega$ :

$$
\begin{aligned}
& \mathrm{S}_{n}(\omega)=\mid \iint \ldots \int \mathrm{d} \Omega_{1} \ldots \mathrm{d} \Omega_{\mathrm{n}-1} \mathrm{E}\left(\Omega_{1}\right) \ldots \\
& \left.\mathrm{E}\left(\Omega_{\mathrm{n}-1}\right) \mathrm{E}\left(\omega-\Omega_{1} \ldots-\Omega_{\mathrm{n}-1)}\right)\right|^{2}
\end{aligned}
$$

where $E(\Omega)=A(\Omega) \exp (\mathrm{i} \phi(\Omega))$ is the Fourier transform of the time-dependent electric field. Interference between components of the fundamental field shapes the nonlinear power spectrum; in particular, $\mathrm{S}_{n}(\omega)$ can be shaped by manipulation of the spectral phase $\phi(\omega)$ alone.

As an example of how the nonlinear power spectrum $\mathrm{S}_{2}(\omega)$ of optimal pulses was identified as a likely control parameter for two photon absorption, we describe results from a recent investigation of saturated absorption in the laser dye LD690. ${ }^{[8]}$ The nonlinear absorption spectrum (NLAS) of LD690, defined by the spectrum of light transmitted through an optically saturated solution, was probed as a function of pulse intensity, spectrum, and spectral phase. When LD690 is excited by pulses on the blue side of the ground state absorption spectrum, the NLAS revealed signatures of excited state absorption and stimulated resonance Raman scattering that depended on pulse chirp. A genetic algorithm was used to identify pulses capable of maximizing and minimizing exited state absorption at $560 \mathrm{~nm}$ (Figure 3, top). Calculation of the 2nd order nonlinear power spectrum $\mathrm{S}_{2}(\omega)$ of optimal pulses showed a correlation between a sharp $S_{2}(\omega)$ feature and increased excited state absorption. Our analysis suggested that the algorithm explored tuning $\mathrm{S}_{2}(\omega)$ to optimize the absorption signal fitness criterion.

Nonlinear power spectra may not provide the most relevant parameterization, and multiphoton control of $S_{n}(\omega)$ does not necessarily correlate highly with fitness. To illustrate this, we describe results from a series of investigations where multiphoton control of the 1,3-cyclohexadiene (CHD) to cis-hexatriene (Z-HT) ring opening reaction in solution was pursued using shaped pulses centered at $800 \mathrm{~nm}$ to launch a wavepacket on the excited electronic states. ${ }^{[52,53]}$ The presence of Z-HT was detected via absorption of an ultraviolet probe. Experimental details may be found in refs. [52] and [53]. The best shaped pulses produced twice as much Z-HT as a transform-limited $800 \mathrm{~nm}$ pulse, with the highest yields of Z-HT occurring for pulses possessing substantial negative quadratic phase. 

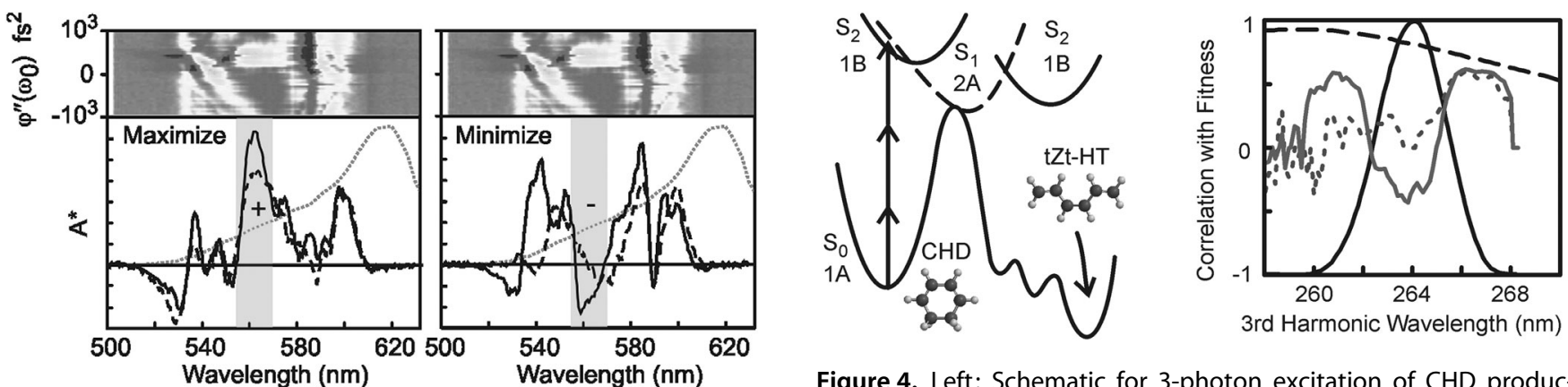
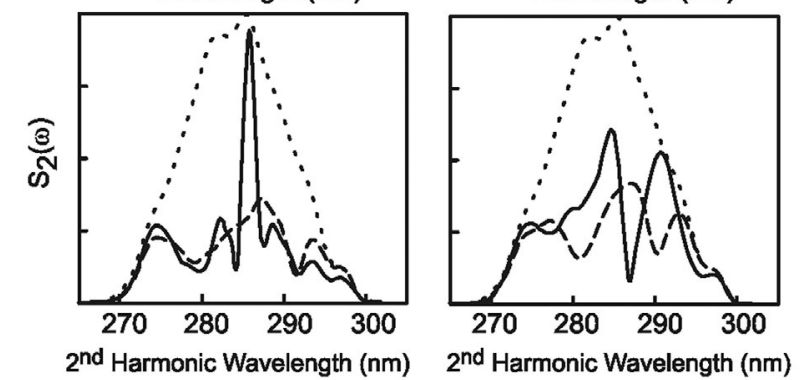

Figure 3. Top: The nonlinear absorption spectrum as a function of linear chirp. White corresponds to maximum absorption, black corresponds to negative absorption (stimulated emission or stimulated Raman), and grey corresponds to little or no absorption or emission. Below these spectra are plotted line-outs of the optimal spectra obtained when the fitness goal was to maximize (left) or minimize (right) the absorption around $560 \mathrm{~nm}$ indicated by the grey shaded region. The dashed lines are the optimal spectra when only the linear chirp was varied $\left(\phi^{\prime \prime}=400 \mathrm{fs}^{2}\right.$ maximizes the absorption and $\phi^{\prime \prime}=-350 \mathrm{fs}^{2}$ minimizes the absorption (i.e., maximizes the emission)). The solid lines are the spectra obtained using optimal pulses identified in GA searches where many pulse parameters could be varied. The dotted lines are the linear ground state absorption spectrum of LD690. Bottom: The second order nonlinear power spectrum $S_{2}(\omega)$ for the optimal pulses using linear chirp alone (dashed lines) and for the GA result (solid lines). The dotted line is the expected $S_{2}(\omega)$ for a transform limited pulse.

Because the coherent control scheme relied on multiphoton absorption to initiate the CHD ring-opening, pulse intensity was expected to be a dominant control parameter for Z-HT production. The probability of threephoton absorption scales as $\int S_{3}(\omega) \mathrm{d} \omega$ or $\int \mathrm{I}^{3}(\mathrm{t}) \mathrm{dt}$ in the wide-linewidth limit. Remarkably, little correlation with peak pump intensity was observed. Non-transform-limited pulses were found to be effective despite the anticipated intensity dependence. ${ }^{[52,53]}$

The data also exhibited little or no correlation between the intensity at any given wavelength in the three-photon spectrum and the fitness or photoproduct signal, as illustrated in Figure 4. In this case, the intuitive parameter reduction turned out not to be the control parameter. This example potentially breaks down the guiding hypothesis ( $\mathrm{I}^{3}$ is known to be a control parameter, but does not correlate with fitness), but it may be a false counterexample if an unidentified control parameter influences fitness in competition with $\mathrm{I}^{3}$.
Figure 4. Left: Schematic for 3-photon excitation of CHD producing cis-hexatriene (tZt-HT). Right: Correlation of fitness with three photon intensity for the excitation of CHD in hexane for two different data sets (solid and dotted grey lines). Zero indicates no correlation with fitness; positive numbers indicate a positive correlation; and negative numbers an anticorrelation with fitness. The solid black line is the calculated 3rd order power spectrum for transform limited pulses. The dashed line is the linear absorption spectrum of $\mathrm{CHD}$ in this region.

\subsubsection{Chirp Space}

Comparison of pulse fitness with peak intensity or specific spectral features can lend insight into potential control mechanisms. In many instances the influence of pulse parameters on the control mechanism may be subtle and require a more detailed analysis of the pulse parameters. In most experiments, the pulse shaping apparatus and search algorithms do not incorporate molecule-specific knowledge. Instead, the search is conducted in a basis determined by experimental convenience, for example the spectral phase at each pixel of the pulse shaper. The resulting search space is typically high-dimensional and difficult to visualize. Reducing the dimensionality of the parameter space can aid in the analysis and interpretation of the pulse fitness.

In the two examples that follow, plotting fitness as a function of projection onto linear and quadratic chirp coefficients lent insight into the nature of the optimization. A Taylor series expansion of the spectral phase provides a useful intuitive representation for many experiments since the low-order terms immediately provide information about time shifts, linear chirp, and quadratic chirp. If pulse phase varies relatively smoothly then the leading terms in the Taylor expansion will dominate the description of the pulses. These variables are used to generate a fitness landscape, representing a map of how fitness might depend on the reduced parameter space.

Two-parameter fitness maps are plotted in the middle and bottom panels of Figure 5 for data obtained from experiments on the ring-opening reaction of CHD in two solvents, cyclohexane and hexane. ${ }^{[53]}$ These solutions were studied because control experiments in neat solvents had revealed that multi-photon interactions in cyclohexane resulted in side reactions with permanent photoproducts absorbing in the same spectral region as Z-HT, including 

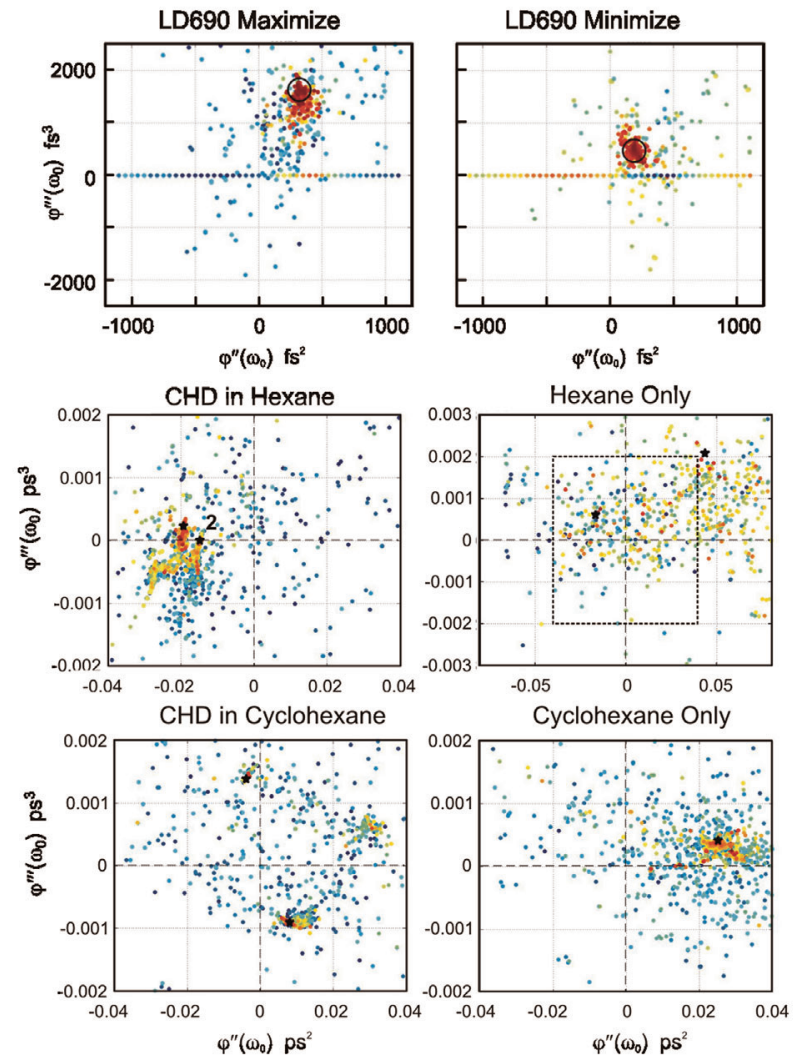

Figure 5. Two-dimensional fitness landscapes parameterized in orders of chirp. Pulse fitness is compared with the projection of spectral phase onto linear and quadratic chirp. Fitness is indicated by color with red corresponding to high fitness and blue corresponding to low fitness. The black circles/stars represent the optimal solutions found in different GA runs. Top: Maximization or minimization of excited state absorption at $560 \mathrm{~nm}$ in LD690. ${ }^{[80]}$ The results of a linear chirp scan are shown as dots along the $x$ axis. Middle: Optimization of photoproduct from CHD in hexane or neat hexane. Two different fitness functions were used in the optimization. The point labeled " 2 " is the optimal solution when a penalty for solvent fragmentation is introduced into the fitness function. ${ }^{[53]}$ Bottom: Optimization of photoproducts from CHD in cyclohexane or neat cyclohexane. The solvent-only search of hexane sampled a much larger region of phase space. The dashed square corresponds to the space plotted in the other three maps.

direct formation of Z-HT from cyclohexane. ${ }^{[52]}$ Such a reaction likely occurs through stepwise hydrogen abstraction.

Comparison of the four fitness maps (each of which represents several independent GA searches) revealed differences in GA convergence and the complexity of the fitness landscape within the chirp parameterization for the different samples. It is apparent that GA searches to optimize Z-HT formation in CHD in hexane converged well to a region of negative linear chirp; high fitness pulses are clustered around the best solutions, whereas the fitness is low in other quadrants of the subspace. Positive chirp is not as effective at optimizing the formation of Z-HT, indicating that the mechanism of control is not simply to reduce peak power or increase pulse duration. In neat hexane, where no multi-photon photochemistry is observed, high fitness pulses are not clustered and the searches do not appear to converge.

In contrast, GA searches converged for both $\mathrm{CHD}$ in cyclohexane and neat cyclohexane samples, but to different regions of the chirp space. Searches in neat cyclohexane consistently converged to large values of positive linear chirp and moderate values of positive quadratic chirp. GA searches for CHD in cyclohexane also exhibit clusters of high fitness pulses, but that repeated searches converge to different regions suggests a more complex underlying parameter space. Additionally, clusters in the region of chirp space optimal for solvent photochemistry suggest that the GA is pressured by the competing solvent reaction. In the face of this complexity, the "optimal" pulse shapes may actually represent compromise solutions for both the CHD ring-opening and the cyclohexane pathway to Z-HT. Alternatively, the different optimal solutions may reflect the influence of a level set describing the range of optimal solutions. ${ }^{[29]}$ There need not be a unique mechanism.

Similarly, fitness landscapes for low orders of chirp were constructed from GA data where the adaptive control experiment sought to optimize excited state absorption in the NLAS of LD690. ${ }^{[80]}$ The fitness landscapes and the fitness evaluated during scans of linear chirp are shown in Figure 5 (Top). The fitness landscapes exhibited clustering similar to the previous example. Comparison of the fitness landscape based on these two data sets shows that the high fitness pulses from the GA search are not well approximated by linear chirp alone. The best GA pulse for maximizing excited state absorption has a quadratic phase coefficient similar to the best linear chirp from the scan, but also has a significant cubic contribution to the phase. Furthermore, minimizing excited state absorption leads to pulses closer to the transform limit. For minimization there are notable differences in the NLAS observed using the best GA pulse compared to linear chirped pulses, particularly on the blue edge of the spectrum (see Figure 3). A scan of third order phase would help determine whether quadratic chirp is actually a control parameter.

\subsubsection{Intuitive Parameter Reduction: A Search Parameterization Inspired By Chirp}

It is also possible to carry out the experimental search itself within an intuitive, reduced-parameter space. For instance, GA searches have been carried out in orders of chirp $^{[29]}$ or by using Legendre polynomials. ${ }^{[27]}$ Here we discuss a particularly intuitive 2-parameter basis used by Vogt et al. to investigate a pump-dump mechanism. ${ }^{[30]}$ They apply a V-shaped phase function across the laser spectrum. The spectral phase is linear up to some point 
(dubbed the spectral break point) at which the sign of the slope of the linear phase is reversed. By adjusting the frequency of the spectral break point and the slope of the linear phase, they create two pulses with different colors and relative delay. Vogt et al. used this parameterization in a GA optimization of the excitation efficiency of IR140. It is known from the work of Shank that linearly chirping the excitation pulse affects excitation efficiency via an intrapulse pump-dump mechanism. ${ }^{[1]}$ Vogt et al. found that colored double pulses can also be used to control the excitation efficiency via the same mechanism. Additionally, since only two parameters are needed, the search space is easily scanned to create a fitness landscape.

\subsection{Multivariate Analysis}

If the pulses are represented by a string of $n$ numbers, then we can consider the pulses to be vectors in an $n$-dimensional vector space, with each vector mapping to a fitness value. We then have a variety of standard statistical techniques available to answer questions about the structure of the space, the nature of the pulses tested, and the relationship between the structure of the space and fitness. Two methods that have been successfully applied to coherent control problems are principle components analysis $(\mathrm{PCA})^{[73]}$ (used in what White et al. dub Principle Control Analysis) and partial least squares regression (PLS) analysis. ${ }^{[72,75]}$ The goal of both of these methods is to find a transformation from the original space to a natural control basis, by which we mean a subspace spanned by a minimum number of vectors needed to define the active control space. In general, a global linear transformation is not adequate to diagonalize the control space. ${ }^{[74]}$ Unfortunately, unguided nonlinear dimension reduction is difficult. When a nonlinear transformation is desired, a common approach is to first transform the data and then use linear methods on the transformed data.

The guiding idea behind both Principal Control Analysis and PLS is to find a linear subspace (or, more properly, an affine subspace) of the search space in which there is both large variation in the genes (or a related parameterization) across the subspace and a substantial correlation with the fitness. Intuitively, these are directions in the search space which were both well-explored and which correlated with fitness.

In principal control analysis, a principal component analysis of the entire collection of genomes is followed by an additional step to determine the correlation of each principal component (PC) with fitness. PCA is a standard statistical technique that finds the best $m$-dimensional linear approximation (in a least-squares sense) to $p$-dimensional data, where $m<p$. The PCA consists of diagonalizing the covariance matrix of the genomes (or some other representation of the pulses from the search). The diagonalization is a unitary transformation to a new basis in which there is no covariance, so that the variance in the data may be explained by examining the variance along individual directions. The eigenvectors of the covariance matrix are referred to as principal components (PCs) and the eigenvalues are the variance of the genomes along each PC direction. The "principal controls" are then those PCs for which both the variance of the data (PC eigenvalue) and the correlation of fitness with projection onto the PC are large.

Principal Control Analysis applied to a number of GA searches in CHD in hexane and cyclohexane identified several features resembling low order polynomial phase (Figure 6). ${ }^{[53]}$ These features exhibited a weak correlation
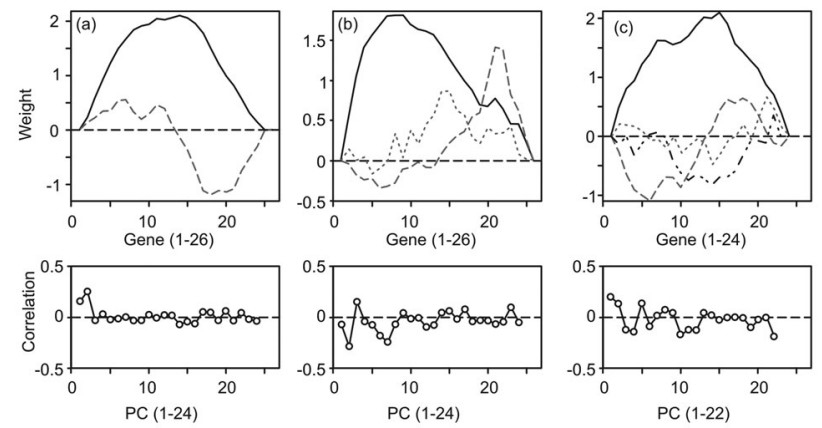

Figure 6. Principle components (PC) for CHD in cyclohexane (left), for neat cyclohexane (center) and for CHD in hexane (right). Solid line $=P C 1$, grey dashed line $=P C 2$, grey dotted line $=P C 3$, black dot-dot-dashed line $=P C 4$. The bottom plots show the correlation of the principle components with fitness.

with fitness, in agreement with the discussion about chirp above. In contrast, negative second-order polynomial phase was uncorrelated with fitness in solvent-only experiments. Principal Control Analysis, while broadly agreeing with the chirp analysis above, also suggests that chirp is not the whole picture - the analysis indicates that other pulse features also play an important role in the formation of Z-HT from CHD in hexane (Figure 6c).

In PLS, both variance and fitness are used together to determine "interesting" directions (although in general PLS calculations tend to be dominated by the variance) ${ }^{\left[{ }^{[2]}\right.}$ Montgomery et al. used PLS to study multiphoton absorption by $\left[\mathrm{Ru}(\mathrm{dpb})_{3}\right]\left(\mathrm{PF}_{6}\right)_{2}$ in solution. ${ }^{[49,72,75]}$ $\left[\mathrm{Ru}(\mathrm{dpb})_{3}\right]\left(\mathrm{PF}_{6}\right)_{2}$ does not absorb at $800 \mathrm{~nm}$, but can be excited through multiphoton absorption. Excitation is detected through spontaneous emission around $640 \mathrm{~nm}$. A beam of phase-shaped pulses at $800 \mathrm{~nm}$ was split, with part of the beam incident on the $\left[\mathrm{Ru}(\mathrm{dpb})_{3}\right]\left(\mathrm{PF}_{6}\right)_{2}$ and part of it going into a $\beta$-barium borate (BBO). GA searches were performed to optimize the ratio of emission to SHG. An analysis of the pulses from the search suggests that control arises from tuning of the two-photon power spectrum towards the red. ${ }^{[49]}$ By applying PLS to the phase profile of the shaped pulses, it was found that 7 
dimensions (of 208) were responsible for $\sim 90 \%$ of the variance of the fitness and that just three dimensions accounted for $82 \%$ of the fitness variance. Searches conducted in this lower-dimensional control basis converged more quickly. ${ }^{[83]}$ Montgomery et al. also implemented a variation of PLS in which they used a single parameter to model the fitness in a local region. To test the local PLS model, single-parameter pulses were evaluated experimentally and their performance was found to be in good agreement with prediction. Furthermore, the singleparameter pulses appeared to be taking advantage of the spectral control mechanism; spectral tuning of the twophoton power spectrum toward the red was apparent in the two-photon spectrum of these pulses. The PLS model appears to have captured the physics responsible for control in this molecule.

Roslund et al. incorporate multivariate analysis into the search itself by using the covariance matrix adaptation variant of the derandomized evolutionary strategy (DES).$^{[84]}$ In the DES, each genome maintains its own set of strategy parameters that determine the likelihood of a mutation in any given direction. As the search progresses, genomes update their strategy parameters by applying PCA to successful mutations. In this way the DES "learns" how fitness correlates with direction. When applied to the problem of shaping $800 \mathrm{~nm}$ pulses to maximize SHG in BBO, the DES converged up to 9 times more rapidly than a bit-string encoded GA. In addition, the local covariance information is available for post facto analysis. In particular, the estimated covariance matrix can be used to estimate the Hessian at an optimum in the fitness landscape. In an experiment using shaped $800 \mathrm{~nm}$ pulses to maximize population transfer from the $5 S \rightarrow 5 P \rightarrow 5 D$ states in atomic rubidium, Roslund et al. used the estimated covariance matrix at the point of maximum yield to estimate the Hessian. The first several eigenvectors of the Hessian (the most yield-sensitive directions) contained structure at wavelengths corresponding to the relevant transitions in $\mathrm{Rb}\left(5 S_{1 / 2} \rightarrow 5 P_{1 / 2} \rightarrow 5 D_{3 / 2}\right.$ and $\left.5 S_{1 / 2} \rightarrow 5 P_{3 / 2} \rightarrow 5 D_{3 / 2,5 / 2}\right)$. These eigenvectors form a reduced-dimensional subspace and could be used as the basis for further investigation.

It is also possible to gain insight from a more traditional PCA analysis. For example, by applying PCA to the best pulses from many GA searches, it is possible to identify features common to the best pulses. The PCs form a low-dimensional subspace approximation to the best pulses. If the dimensionality is low enough, such a subspace can be exhaustively scanned. These ideas were recently applied to a simulated pump-probe control experiment in molecular iodine. ${ }^{[28]}$ In the simulation, a shaped $27 \mathrm{fs}, 550 \mathrm{~nm}$ pump pulse launched a wavepacket onto the B state. A $225 \mathrm{~nm}$ probe at a fixed delay then transferred population from the B to the E state. The goal was to maximize population transfer to the E state. PCA applied to the electric fields of the best pulses from 55 runs indicated that the best solutions could be represented as superpositions of two electric fields. In the time domain, the two fields contained amplitude at the only two times that would result in the launched wavepacket overlapping with the probe pulse. By only varying the relative amplitude and phase of these two basis fields, it was possible to model the best pulses using only two parameters. An exhaustive scan of this two-parameter space confirmed that the model successfully captured the essential physics of the best pulses.

\subsection{Chemical Modification}

The methods described above involve analysis of data sets obtained on a single system, searching for control parameters optimized for a given fitness function. Information can also be extracted from comparison of chemically similar systems. The comparison of the cyclohexadiene ring opening reaction in hexane and cyclohexane provides one simple example of such a comparison. The differences observed between CHD ring-opening in hexane and cyclohexane have the potential to provide insight into possible control mechanisms.

These experiments on CHD involved separate optimization runs in each system, which were compared after the experiments were completed. Herek and coworkers have described a systematic approach where the fitness path optimized in one system is retraced in similar systems to extract information on the molecular mechanism and the influence of external parameters on the mechanism. ${ }^{[85]}$

The method proposed by van der Walle et al. was illustrated using a simple test case involving excitation of Coumarin 6 in both polar and nonpolar solvents. ${ }^{[85]}$ The feedback signal for fitness was generated using the stimulated emission of the dye between $510 \mathrm{~nm}$ and $595 \mathrm{~nm}$, $1 \mathrm{~ns}$ after excitation. The stimulated emission signal is a probe of the magnitude of the excited state population produced by the pump pulse. The fitness landscape was found to be the same in the nonpolar solvents, but distinctly different in polar solvents. In this case the mechanism was known from the outset - arising from the solvent shift of the absorption and emission spectra. The same method, however, could be used in more complex systems to unravel the molecular mechanism for control of chemical reactions.

\section{Summary and Outlook}

Although learning algorithms are now commonly used to find control pulses, the pulse features responsible for control and the mechanism of control often remain unclear. In this paper we have reviewed how the interpretation of coherent control experiments can be enhanced by making use of information contained in the entire collection of 
pulse shapes evaluated during the course of a coherent control experiment. Some of the techniques are physically motivated (such as examining nonlinear power spectra and chirp parameters) while others aim to model the space and identify important features with a minimum of a priori knowledge (multivariate statistical techniques). While these methods are not a substitute for physical intuition, they enhance physical intuition by aiding in visualization and the identification of potentially interesting pulse features.

\section{Acknowledgments}

This work has been supported by the National Science Foundation through Grant No. CHE-0718219, and through the FOCUS Center at the University of Michigan.

\section{References}

[1] M. A. Montgomery, E. M. Grumstrup, N. H. Damrauer, J. Opt. Soc. Am. B 2010, 27, 2518-2533.

[2] J. P. Ogilvie, K. J. Kubarych in Advances in Atomic, Molecular, and Optical Physics, Vol 57 (Eds.: E. Arimondo, P. R. Berman, C. C. Lin), Elsevier Academic Press Inc, San Diego, 2009, pp. 249-321.

[3] E. M. Grumstrup, S. H. Shim, M. A. Montgomery, N. H. Damrauer, M. T. Zanni, Opt. Express 2007, 15, 1668116689.

[4] S.-H. Shim, M. T. Zanni, Phys. Chem. Chem. Phys. 2009, 11, $748-761$

[5] H. Rabitz, R. de Vivie-Riedle, M. Motzkus, K.-L. Kompa, Science 2000, 288, 824-828.

[6] S. A. Rice, S. P. Shah, Phys. Chem. Chem. Phys. 2002, 4, $1683-1700$

[7] M. Shapiro, P. Brumer, Adv. At. Mol. Opt. Phys. 2000, 42, 287-345.

[8] M. Shapiro, P. Brumer, Rep. Prog. Phys. 2003, 66, 859-942.

[9] B. J. Sussman, D. Townsend, M. Y. Ivanov, A. Stolow, Science 2006, 314, 278-282.

[10] M. Roth, L. Guyon, J. Roslund, V. Boutou, F. Courvoisier, J.-P. Wolf, H. Rabitz, Phys. Rev. Lett. 2009, 102, 253001.

[11] M. Kotur, T. Weinacht, B. J. Pearson, S. Matsika, J. Chem. Phys. 2009, 130, 134311.

[12] B. J. Pearson, T. C. Weinacht, Opt. Express 2007, 15, 43854388.

[13] K.-C. Tang, A. Rury, M. B. Orozco, J. Egendorf, K. G. Spears, R. J. Sension, J. Chem. Phys. 2011, 134, 104503.

[14] K.-C. Tang, R. J. Sension, Faraday Discuss. 2011, 153, $117-$ 129.

[15] J. Möhring, T. Buckup, C. S. Lehmann, M. Motzkus, J. Opt. Soc. Am. B 2009, 26, 1538-1544.

[16] M. Hacker, G. Stobrawa, R. Sauerbrey, T. Buckup, M. Motzkus, M. Wildenhain, A. Gehner, Appl. Phys. B 2003, 76, 711-714.

[17] M. Greenfield, S. D. McGrane, D. S. Moore, J. Phys. Chem. A 2009, 113, 2333-2339.

[18] R. S. Judson, H. Rabitz, Phys. Rev. Lett. 1992, 68, 15001503.
[19] C. Brif, R. Chakrabarti, H. Rabitz, New J. Phys. 2010, 12, 075008.

[20] A. Assion, T. Baumert, M. Bergt, T. Brixner, B. Kiefer, V. Seyfried, M. Strehle, G. Gerber, Science 1998, 282, 919-922.

[21] R. J. Levis, H. A. Rabitz, J. Phys. Chem. A 2002, 106, 64276444.

[22] B. J. Pearson, J. L. White, T. C. Weinacht, P. H. Bucksbaum, Phys. Rev. A 2001, 63, 063412.

[23] H. Rabitz, W. S. Zhu, Acc. Chem. Res. 2000, 33, 572-578.

[24] B. Kohler, J. L. Krause, F. Raksi, K. R. Wilson, V. V. Yakovlev, R. M. Whitnell, Y. Yan, Acc. Chem. Res. 1995, 28, 133140.

[25] C. J. Bardeen, V. V. Yakovlev, K. R. Wilson, S. D. Carpenter, P. M. Weber, W. S. Warren, Chem. Phys. Lett. 1997, 280, 151-158.

[26] F. Langhojer, D. Cardoza, M. Baertschy, T. Weinacht, $J$. Chem. Phys. 2005, 122, 014102.

[27] A. Jafarpour, J. Savolainen, R. de Jong, J. Middag, D. P. Sprunken, P. van der Walle, D. Yang, J. L. Herek, Opt. Express 2009, 17, 11986-12000.

[28] J. L. White, D. M. Cardoza, P. H. Bucksbaum, Unpublished work.

[29] J. Roslund, M. Roth, H. Rabitz, Phys. Rev. A 2006, 74, 043414.

[30] G. Vogt, P. Nuernberger, R. Selle, F. Dimler, T. Brixner, G. Gerber, Phys. Rev. A 2006, 74, 033413.

[31] M. Bergt, T. Brixner, C. Dietl, B. Kiefer, G. Gerber, J. Organomet. Chem. 2002, 661, 199-209.

[32] M. Bergt, T. Brixner, B. Kiefer, M. Strehle, G. Gerber, J. Phys. Chem. A 1999, 103, 10381-10387.

[33] D. Cardoza, M. Baertschy, T. Weinacht, J. Chem. Phys. 2005, 123, 074315.

[34] N. H. Damrauer, C. Dietl, G. Krampert, S. H. Lee, K. H. Jung, G. Gerber, Eur. Phys. J. D 2002, 20, 71-76.

[35] R. J. Levis, G. M. Menkir, H. Rabitz, Science 2001, 292, 709-713.

[36] C. Trallero-Herrero, D. Cardoza, T. C. Weinacht, J. L. Cohen, Phys. Rev. A 2005, 71, 013423.

[37] D. Cardoza, M. Baertschy, T. Weinacht, Chem. Phys. Lett. 2005, 411, 311-315.

[38] D. Cardoza, F. Langhojer, C. Trallero-Herrero, O. L. A. Monti, T. Weinacht, Phys. Rev. A 2004, 70, 053406.

[39] D. Cardoza, B. J. Pearson, M. Baertschy, T. Weinacht, J. Photochem. Photobiol. A 2006, 180, 277-281.

[40] C. Daniel, J. Full, L. Gonzalez, C. Kaposta, M. Krenz, C. Lupulescu, J. Manz, S. Minemoto, M. Oppel, P. Ros endoFrancisco, S. Vajda, L. Woste, Chem. Phys. 2001, 267, 247260.

[41] C. J. Bardeen, Q. Wang, C. V. Shank, Phys. Rev. Lett. 1995, $75,3410-3413$.

[42] C. J. Bardeen, V. V. Yakovlev, J. A. Squier, K. R. Wilson, J. Am. Chem. Soc. 1998, 120, 13023-13027.

[43] T. Brixner, N. H. Damrauer, P. Niklaus, G. Gerber, Nature 2001, 414, 57-60.

[44] J. L. Herek, W. Wohlleben, R. J. Cogdell, D. Zeidler, M. Motzkus, Nature 2002, 417, 533-535.

[45] V. I. Prokhorenko, A. M. Nagy, S. A. Waschuk, L. S. Brown, R. R. Birge, R. J. D. Miller, Science 2006, 313, 1257-1261.

[46] A. C. Florean, E. C. Carroll, K. G. Spears, R. J. Sension, P. H. Bucksbaum, J. Phys. Chem. B 2006, 110, 20023-20031.

[47] V. I. Prokhorenko, A. M. Nagy, R. J. D. Miller, J. Chem. Phys. 2005, 122, 184502.

[48] A. Kahan, O. Nahmias, N. Friedman, M. Sheves, S. Ruhman, J. Am. Chem. Soc. 2007, 129, 537-546. 
[49] M. A. Montgomery, N. H. Damrauer, J. Phys. Chem. A 2007, 111, 1426-1433.

[50] O. Nahmias, O. Bismuth, O. Shoshana, S. Ruhman, J. Phys. Chem. A 2005, 109, 8246-8253.

[51] S. Zhang, Z. R. Sun, X. Y. Zhang, Y. Xu, Z. G. Wang, Z. Z. $\mathrm{Xu}, \mathrm{R}$. X. Li, Chem. Phys. Lett. 2005, 415, 346-350.

[52] E. C. Carroll, B. J. Pearson, A. C. Florean, P. H. Bucksbaum, R. J. Sension, J. Chem. Phys. 2006, 124, 114506.

[53] E. C. Carroll, J. L. White, A. C. Florean, P. H. Bucksbaum, R. J. Sension, J. Phys. Chem. A 2008, 112, 6811-6822.

[54] S.-H. Lee, K.-H. Jung, J. H. Sung, K.-H. Hong, C. H. Nam, J. Chem. Phys. 2002, 117, 9858-9861.

[55] G. Vogt, G. Krampert, P. Niklaus, P. Nuernberger, G. Gerber, Phys. Rev. Lett. 2005, 94, 068305.

[56] J. B. Gong, P. Brumer, Ann. Rev. Phys. Chem. 2005, 56, $1-$ 23.

[57] R. J. Gordon, S. A. Rice, Ann. Rev. Phys. Chem. 1997, 48, 601-641.

[58] S. A. Rice, M. Zhao, Optical Control of Molecular Dynamics, John Wiley \& Sons, NY, 2000.

[59] M. Shapiro, P. Brumer, Principles of the Quantum Control of Molecular Processes, Wiley-Interscience, 2003

[60] D. J. Tannor, Introduction to Quantum Mechanics: A Time Dependent Perspective, University Science Books, 2006.

[61] P. Brumer, M. Shapiro, Ann. Rev. Phys. Chem. 1992, 43, $257-282$

[62] R. J. Gordon, L. C. Zhu, T. Seideman, Acc. Chem. Res. 1999, 32, 1007-1016.

[63] D. J. Tannor, S. A. Rice, Adv. Chem. Phys. 1988, 70, 441523.

[64] J. Roslund, M. Roth, L. Guyon, V. Boutou, F. Courvoisier, J.-P. Wolf, H. Rabitz, J. Chem. Phys. 2011, 134, 034511.

[65] J. Petersen, R. Mitric, V. Bonacic-Koutecky, J. P. Wolf, J. Roslund, H. Rabitz, Phys. Rev. Lett. 2010, 105, 073003.

[66] C. J. Bardeen, V. V. Yakovlev, J. A. Squier, K. R. Wilson, S. D. Carpenter, P. M. Weber, J. Biomed. Opt. 1999, 4, $362-$ 367.

[67] V. V. Lozovoy, I. Pastirk, K. A. Walowicz, M. Dantus, J. Chem. Phys. 2003, 118, 3187-3196.

[68] J. P. Ogilvie, D. Débarre, X. Solinas, J.-L. Martin, E. Beaurepaire, M. Joffre, Opt. Express 2006, 14, 759-766.
[69] K. A. Walowicz, I. Pastirk, V. V. Lozovoy, M. Dantus, J. Phys. Chem. A 2002, 106, 9369-9373.

[70] T. Brixner, N. H. Damrauer, B. Kiefer, G. Gerber, J. Chem. Phys. 2003, 118, 3692-3701.

[71] J. P. Ogilvie, K. J. Kubarych, A. Alexandrou, M. Joffre, Opt. Lett. 2005, 30, 911-913.

[72] M. A. Montgomery, R. R. Meglen, N. H. Damrauer, J. Phys. Chem. A 2007, 111, 5126-5129.

[73] J. L. White, B. J. Pearson, P. H. Bucksbaum, J. Phys. B 2004, 37, L399-405.

[74] D. Cardoza, C. Trallero-Herrero, F. Langhojer, H. Rabitz, T. Weinacht, J. Chem. Phys. 2005, 122, 124306.

[75] M. A. Montgomery, R. R. Meglen, N. H. Damrauer, J. Phys. Chem. A 2006, 110, 6391-6394.

[76] J. C. Shane, V. V. Lozovoy, M. Dantus, J. Phys. Chem. A 2006, 110, 11388-11391.

[77] P. van der Walle, H. Offerhaus, J. Herek, A. Jafarpour, Opt. Express 2010, 18, 973-987.

[78] Z. Zheng, A. M. Weiner, Chem. Phys. 2001, 267, 161-171.

[79] M. A. Montgomery, N. H. Damrauer, New J. Phys. 2009, 11, 105053.

[80] E. C. Carroll, A. C. Florean, P. H. Bucksbaum, K. G. Spears, R. J. Sension, Chem. Phys. 2008, 350, 75-86.

[81] G. Cerullo, C. J. Bardeen, Q. Wang, C. V. Shank, Chem. Phys. Lett. 1996, 262, 362-368.

[82] R. T. Trevor Hastie, Jerome Friedman, The Elements of Statistical Learning: Data Mining, Inference, and Prediction, Springer-Verlag, 2001.

[83] M. A. Montgomery, R. R. Meglen, N. H. Damrauer, in Ultrafast Phenomena $X V$ (Eds.: P. Corkum, D. Jonas, R. J. D. Miller, A. M. Weiner), Springer, Berlin, 2006.

[84] J. Roslund, O. M. Shir, T. Back, H. Rabitz, Phys. Rev. A 2009, 80, 043415.

[85] P. van der Walle, J. Savolainen, L. Kuipers, J. L. Herek, Chem. Phys. Lett. 2009, 483, 164-167.

Received: September 14, 2011

Accepted: October 20, 2011

Published online: March 7, 2012 